\title{
BREAST CANCER SURVIVAL: A HOSPITAL REGISTRY-BASED RETROSPECTIVE COHORT OF SÃO PAULO, BRAZIL
}

\author{
Stela V. Peres ${ }^{1,2}$, Alexandre M. Ab'Saber ${ }^{1,3}$, José Eluf Neto ${ }^{1,4}$ \\ ${ }^{1}$ Fundação Oncocentro de São Paulo - São Paulo (SP), Brazil. \\ ${ }^{2}$ AC Camargo Cancer Center - São Paulo (SP), Brazil. \\ ${ }^{3}$ Hospital das Clínicas, Universidade de São Paulo - São Paulo (SP), Brazil. \\ ${ }^{4}$ Departamento de Medicina Preventiva, Faculdade de Medicina, Universidade de São Paulo - São Paulo (SP), Brazil.
}

Objectives: To describe sociodemographic characteristics, clinical stage at diagnosis, treatment, immunohistochemistry subtypes (Estrogen Receptor - ER, Progesterone Receptor - PR, and Human Epidermal growth factor Receptor-type 2 HER2), and survival rates of breast cancer for women treated in São Paulo (Brazil). Methodology: We conducted a hospital registry-based, retrospective cohort study on 907 women with breast cancer, ages ranging from 22 to 96 years old, diagnosed between January 1, 2000 and December 31, 2013. Data were extracted from the Oncocenter Foundation of the State of São Paulo (FOSP). A 5-year overall survival analysis was performed using the Kaplan-Meier method and Cox proportional hazard ratios (HR) and 95\% confidence interval (95\%CI) were calculated. Results: Mean age at diagnosis was 57 years (DP=13.2). According to histology, 74\% were invasive ductal carcinoma and 63.5\% were classified as I or II (63.4\%). For treatment, 51.4\% were combined therapy (surgery/RT/QT/HT). ER+/PR+/HER2- was the most common subtype (528; $58.2 \%)$. In the multiple analysis, subtype $\mathrm{EP}+\mathrm{RP}+\mathrm{HER} 2-\left(\mathrm{HR}_{\mathrm{adj}}=0.43 ; \mathrm{p}<0.001\right)$ and combined therapy $\left(\mathrm{HR}_{\mathrm{adj}}=0.62 ; \mathrm{p}=0.014\right)$ were both associated with a reduction in the risk of death. For clinical stage at diagnosis, the stages III and IV have shown an increased risk of death $\left(\mathrm{HR}_{\mathrm{adj}}=2.87 ; \mathrm{p}<0.001\right.$ and $\mathrm{HR}_{\mathrm{adj}}=10.43 ; \mathrm{p}<0.001$, respectively). Conclusions: In our study, combined treatment and $\mathrm{EP}+\mathrm{RP}+/ \mathrm{HER} 2$ - subtype were predictive prognostic factors for high overall survival rates in women with invasive breast cancer. On the other hand, advanced clinical stage (III/IV) at diagnosis have shown as unfavorable prognosis for overall survival rates. 